\title{
Novel Frequency-Selective Rasorber with Ultrawide Absorption Bandwidth Covering Both the $X$ - and Ku-Bands
}

\author{
Jie Xiong ${ }^{1,2}$, Yanjie Wu ${ }^{1}$, Yu Mao ${ }^{1}$, Feng Deng ${ }^{3}$, Lijie Chen ${ }^{3}$ and Hai Lin ${ }^{1, *}$ \\ 1 College of Physics and Science and Technology, Central China Normal University, Wuhan 430079, China; \\ xiongjie82@mails.ccnu.edu.cn (J.X.); wuyanjie@mails.ccnu.edu.cn (Y.W.); pistachio@mails.ccnu.edu.cn (Y.M.) \\ 2 Institute of Physics and Telecommunications, HuangGang Normal University, Huanggang 438000, China \\ 3 Science and Technology on Electromagnetic Compatibility Laboratory, China Ship Development and Design Centre, \\ Wuhan 430000, China; dengfengzc@gmail.com (F.D.); lijiechen1985@gmail.com (L.C.) \\ * Correspondence: linhai@mail.ccnu.edu.cn; Tel.: +86-186-2788-4302
}

Received: 5 August 2020; Accepted: 2 September 2020; Published: 5 September 2020

\begin{abstract}
A novel dual-polarized transmissive/absorptive frequency-selective rasorber (FSR) with an ultrawide absorption spectrum covering both the $\mathrm{X}$ - and $\mathrm{Ku}$-bands is proposed in this paper. The FSR is constructed from a bottom lossless transmission layer and a top lossy absorption layer, in which a resistor-loaded incurved square loop strip line structure is utilized to obtain an ultrawide absorption band. To quantitatively analyze its operation principle, an accurate equivalent circuit model of the proposed FSR was developed. A 2D prototype was designed, assembled, fabricated, and measured. The FSR exhibits an absorption band that ranges from 8.1 to $19.1 \mathrm{GHz}(81 \%)$ under normal incidence, whereas the passband insertion loss at $4.5 \mathrm{GHz}$ is less than $0.45 \mathrm{~dB}$. The total thickness of the FSR is only $5.1 \mathrm{~mm}$, which keeps low profile characteristics. The simulation agrees well with the measured results.
\end{abstract}

Keywords: frequency-selective rasorber; ultrawide absorption; $\mathrm{X}$ and Ku-band; dual-polarized

\section{Introduction}

A frequency-selective surface (FSS) is regarded as a spatial filter that can tailor the frequency response of incident electromagnetic waves [1-4]. Since FSS can reflect the out-of-band incident wave to other directions while remaining transparent for in-band signals, it has been widely used in radar or antenna systems as radomes to reduce the radar cross section or suppress interference [5,6]. However, the FSS can only deal with mono-static Radar Cross Section (RCS) reduction, and arbitrary reflected out-of-band signals will lead to an increase of bistatic RCS [7-9]. To solve this imperfection, a so-called frequency-selective rasorber (FSR) was proposed [10] and has already attracted much attention [11-23]. The operation mechanism of an FSR is a combination of traditional FSS and a metamaterial absorber; it thus absorbs the out-of-band waves instead of reflecting them to reduce the bistatic RCS.

An ideal FSR should have zero insertion loss (IL) for operating in-band electromagnetic (EM) signals while having $100 \%$ absorptivity for out-of-band signals [11-14]. To achieve these goals, both 2D-FSR and 3D-FSR have been developed in previously published studies [11-26]. The 2D-FSR maintains the multilayer structural form similar to FSS, and it usually consists of a lossy resistive sheet and a lossless FSS layer [14,15]. The lossy layer performs as an absorber for out-of-band signals while the lossless layer stops the undesired signal and allows the in-band signal to transmit freely. The unit cell of a 3D-FSR is usually constructed from several pairs of parallel-plate PCB boards to form multimode cavities [15-18]. These cavities contain a lossy mode and a lossless mode for different frequency band EM waves. Due to its three-dimensional structure, 3D-FSR is normally difficult to 
fabricate, and is also thicker than its 2D counterpart, which makes it difficult to conform with the configuration of the antenna system.

Currently, according to the relative locations between the passband and the absorption band, FSRs can be divided into three basic types: absorption band above the passband; absorption band below the passband; and absorption (pass)band inside pass (absorption) band [25,26]. Different types of FSRs should meet individual requirements under different application scenarios. For instance, the most severe threat faced by military vessels comes from missiles and fighters whose fire control radar systems normally work on the $\mathrm{X}$ and Ku bands; thus, the FSR radomes for the searching radar systems on the vessels should have an ultrawide absorption band covering both the $\mathrm{X}$ and $\mathrm{Ku}$ bands while allowing the $C$ band radar signal to pass through. Since the lossy layer of the 2D-FSR may induce insertion loss, it is not trivial to simultaneously satisfy all of the designated needs for this kind of FSR: dual polarization, ultrawideband absorption and insertion loss below $1 \mathrm{~dB}$. Extending the absorptive bandwidth and lowering the insertion loss in the passband are two main aspects for the design of transmissive/absorptive FSR. Several research groups are currently trying to achieve the best performance in such a device. As shown in [19], the Jerusalem cross and the double square loops with loaded resistors were selected as the bandpass FSS and the resistive FSS, respectively. The radome obtains an absorptive band ranging from 5.3 to $14.8 \mathrm{GHz}$. In another work [20], the isolation loss of the FSR reached $0.76 \mathrm{~dB}$, whereas the absorption band was within the range of 10.6 to $18 \mathrm{GHz}$. In addition, several miniaturized FSRs with broad absorption bandwidth were also reported in [21-24], while the isolated loss of these designs was still higher than $1 \mathrm{~dB}$.

In this paper, we focus on the design of an 2D-FSR with an absorption band above the passband, whose absorption band covers both the $\mathrm{X}$ - and Ku-bands; in other words, its $-10 \mathrm{~dB}$-absorption band ranges between 8.1-19.1 GHz; the FSR's passband is located in the $\mathrm{C}$ band, at $4.5 \mathrm{GHz}$, and achieves an insertion loss as low as $0.45 \mathrm{~dB}$. The FSR also works for EM waves with both transverse electric (TE) and transverse magnetic (TM) polarization.

\section{Structure and Simulation Results}

The proposed FSR is illustrated in Figure 1a. It consists of a lossy layer on top of a lossless FSS layer; the two layers are separated from each other by an air gap with a thickness of $3.5 \mathrm{~mm}$. The unit cells of the FSR are printed on a $0.8 \mathrm{~mm}$-thick Rogers $4003 \mathrm{C}$ substrate $(\varepsilon=3.55)$. The unit cell on the upper layer is designed as an incurved square loop loaded with four resistors on its four arms, and the resistance is $127 \Omega$. The lower frequency-selection layer consists of a modified Jerusalem cross aperture array, where the four arms of the cross-slots are replaced with meanderline slots. All of the key parameters of the FSR are listed in Table 1. In order to qualitatively analyze the operating principle, an equivalent circuit model (ECM) of the FSR is introduced and is shown in Figure 1d. According to the transmission line theory, the two parallel branches $Z_{1}$ and $Z_{3}$ represent the impedance of the lossy layer and lossless layer, respectively. The transmission line section $\left(Z_{2}, \theta\right)$ represents the hybrid path, which is comprised of impedance of the substrate and impedance of the air between the two-layer substrate. From the principle of a conventional impedance converter, the electric length $\theta$ is proposed to be $\lambda / 4$ ( $\lambda$ is the free-space wavelength). As illustrated in Figure $1 d, L_{1}, L_{2}$, and $L_{3}$ represent the inductance of different parts of the incurved square loop. $C_{1}, C_{2}, C_{3}$, and $C_{5}$ represent the capacitors of the corresponding electric fields in different parts. $R_{1}$ is the lumped resistance in the model. For the lossless FSS layer, under the radiation of incident electromagnetic waves, the Jerusalem cross aperture array appears as a parallel LC resonator $\left(\mathrm{L}_{4}\right.$ and $\left.\mathrm{C}_{4}\right)$ with passband characteristics. All parameters of the ECM of our proposed FSR are listed in the caption of Figure 1. 


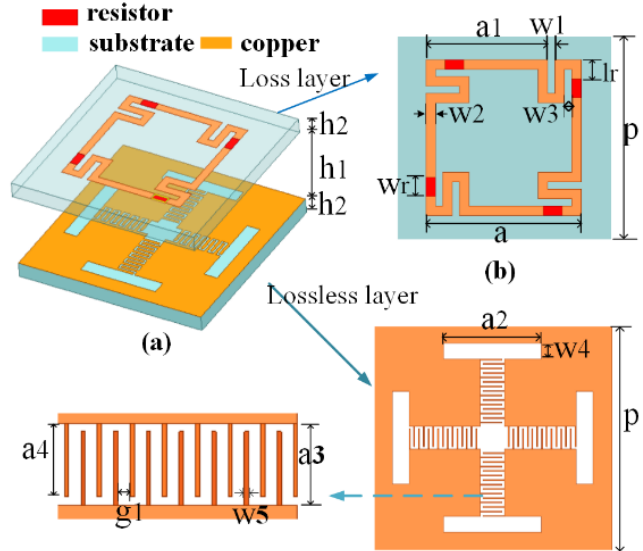

(c)

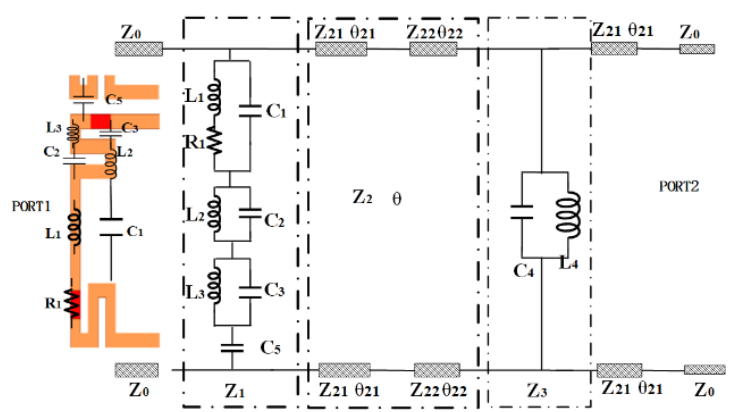

(d)

Figure 1. (a) 3D view of the cascaded FSS; (b) loss layer; (c) lossless layer; (d) equivalent circuit of FSR. (The circuit parameters: $Z_{0}=377 \Omega, L_{1}=1.85 \mathrm{nH}, C_{1}=0.04 \mathrm{pF}, L_{2}=L_{3}=0.29 \mathrm{nH}, C_{2}=C_{3}=0.21 \mathrm{pF}$, $L_{4}=1.66 \mathrm{nH}, C_{4}=0.75 \mathrm{pF}, C_{5}=0.06 \mathrm{pF}, R_{1}=127 \Omega, Z_{21}=500 \Omega, \theta_{21}=25$ at $13.5 \mathrm{GHz}, Z_{22}=80 \Omega$, $\theta_{22}=63$ at $\left.13.5 \mathrm{GHz}\right)$.

Table 1. Unit cell dimensions of FSR layers (mm).

\begin{tabular}{ccccccccccccccccc}
\hline $\mathbf{p}$ & $\mathbf{a 1}$ & $\mathbf{a} 2$ & $\mathbf{a 3}$ & $\mathbf{a 4}$ & $\mathbf{w 1}$ & $\mathbf{w} \mathbf{2}$ & $\mathbf{w 3}$ & $\mathbf{w 4}$ & $\mathbf{w 5}$ & $\mathbf{g 1}$ & $\mathbf{a}$ & $\mathbf{h 1}$ & $\mathbf{h} \mathbf{2}$ & $\mathbf{w r}$ & $\mathbf{l r}$ & Resistor \\
\hline 10 & 4.8 & 3.9 & 1 & 0.9 & 0.1 & 0.35 & 0.1 & 0.5 & 0.05 & 0.15 & 5.7 & 3.5 & 0.8 & 0.8 & 0.8 & $127 \Omega$ \\
\hline
\end{tabular}

To meet the designated requirements for real application, the center frequency of the passband was set to $4.5 \mathrm{GHz}$, and the absorption band should cover both the $\mathrm{X}$ - and $\mathrm{Ku}$-bands. Thus, for the ideal case, $\left|S_{11}\right|$ of the FSR should be equal to zero and the $\left|S_{21}\right|$ should be equal to 1 at $4.5 \mathrm{GHz}$, while both $\left|S_{11}\right|$ and $\left|S_{21}\right|$ are equal to zero within the absorption band.

The ABCD matrix of the FSR can be written as [7]:

$$
\left[\begin{array}{ll}
\mathrm{A} & \mathrm{B} \\
\mathrm{C} & \mathrm{D}
\end{array}\right]=\left[\begin{array}{cc}
1 & 0 \\
1 / \mathrm{Z}_{1} & 1
\end{array}\right]\left[\begin{array}{cc}
\cos \theta & j \mathrm{Z}_{2} \sin \theta \\
j\left(1 / Z_{2} \sin \theta\right) & \cos \theta
\end{array}\right]\left[\begin{array}{cc}
1 & 0 \\
1 / Z_{3} & 1
\end{array}\right]
$$

where $\theta$ is the electric length of hybrid path. Generally, the value of $Z_{2}$ is selected to be the same as $Z_{0}$ for the purpose of a simplified calculation. Then, its transmission coefficient $\left(S_{21}\right)$ can be calculated as follows:

$$
\left|s_{21}\right|=\frac{2}{\left|A+\frac{B}{Z_{0}}+C Z_{0}+D\right|}=\frac{2}{\left|\left(2+\frac{Z_{0}\left(Z_{1}+Z_{3}\right)}{Z_{1} Z_{3}}\right) \cos \theta+j\left(2+\frac{Z_{0}\left(Z_{1}+Z_{3}+Z_{0}\right)}{Z_{1} Z_{3}}\right) \sin \theta\right|}
$$

Since $Z_{0}$ is the wave impedance of free space, which is $377 \Omega$, the value $\left|S_{21}\right|$ is mainly determined by $Z_{1}$ and $Z_{3}$. To satisfy the requirements that $\left|S_{21}\right|=1$ at a passband of $4.5 \mathrm{GHz}$, when $z_{3} \rightarrow \infty$ or $z_{1} \rightarrow \infty$, the transmission coefficient can be simplified as follows:

$$
\left|S_{21}\right|=\frac{2}{|2(\cos \theta+\sin \theta)|}
$$

According to the circuit theory, under parallel resonance, the impedance of the resonator turns to infinite, and when the parameters are properly chosen, the condition $\left|S_{21}\right|=1$ can be satisfied. 
The resonant frequency of the bottom FSS should be assigned in the passband that is determined by the parallel $\mathrm{LC}$ resonator $\left(\mathrm{L}_{4}, \mathrm{C}_{4}\right)$ in the ECM.

$$
f_{1}=\frac{1}{2 \pi \sqrt{L_{4} C_{4}}}=4.5 \mathrm{GHz}
$$

Since the in-band signals pass through the lossy layer and lossless layer, the IL is unavoidable due to the lossy elements. The key to realize a low IL FSR depends on the design of the lossy resistive layer. The equivalent impedance of the lossy layer should be infinite to obtain a transmission coefficient near unity. According to the previous analysis, a parallel resonance LC structure can be used to form infinite impedance; we thus designed an incurved structure to construct a parallel $\mathrm{LC}\left(\mathrm{L}_{2} / / \mathrm{C}_{2}, L_{3} / / C_{3}\right)$ to reduce the insertion loss in the passband.

When the FSR works in the absorbing band, as the absorbing band is far away from the resonant frequency of the passband, the lossless layer is almost non-resonant in the absorbing band, and it can satisfy $Z_{3}=0$ in the absorbing band. To design a wider absorption band, two resonance frequencies were realized by designing two LC parallel circuits and two LC series circuits. At these two frequencies (9.4 and $17.4 \mathrm{GHz}), Z_{1}$ gets cancelled with $Z_{2}$, resulting in a double valley low reflectivity characteristic.

The proposed FSR's unit cell was simulated by the full wave numerical simulation software (HFSS), and the ECM was simulated by a circuit simulator Advanced Design System 2019 (ADS2019). The simulation results are shown in Figure 2a; it can be seen that the full wave simulation results agreed well with the circuit simulation results in the entire frequency band. The absorptivity of the FSR is shown separately in Figure $2 b$, it can be seen from Figure $2 b$ that the proposed FSR exhibited $a$ wideband-absorption property and that the fractional bandwidth (FBW) of the $10 \mathrm{~dB}$-absorption band was $81 \%$ (from 8.1 to $19.1 \mathrm{GHz}$ ) with an absorptivity greater than $90 \%$; at the same time, the in-band IL approached $0.45 \mathrm{~dB}$.

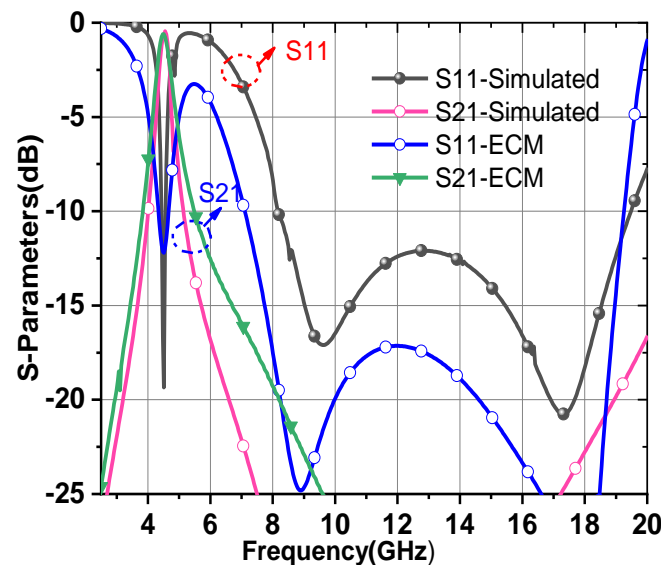

(a)

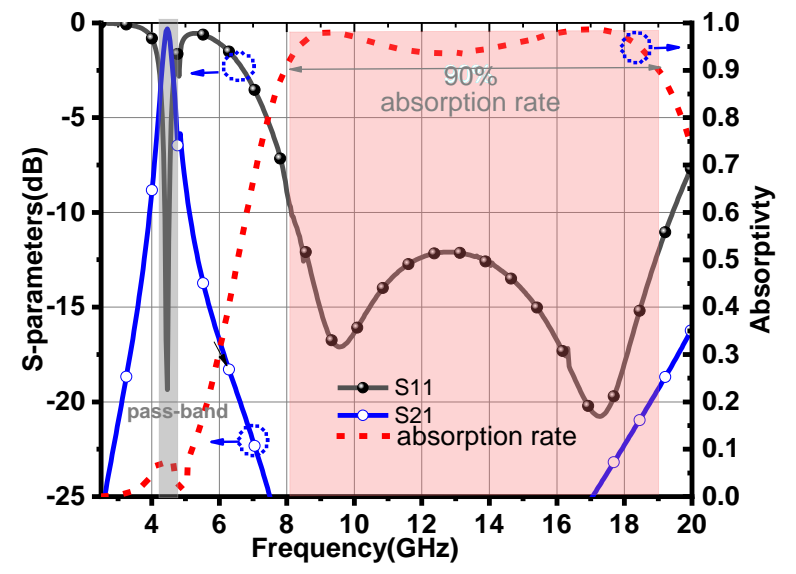

(b)

Figure 2. The $\mathrm{S}$ parameter of the ECM results and simulation results under normal incidence. (a) The $\mathrm{S}$ parameter of the ECM results and simulation results; (b) absorption rate of FSR.

To obtain an optimal absorption performance of the FSR with minimum insertion loss, the resistance value $R_{1}$ and structure parameter $\mathrm{a}_{1}$ are two key parameters for the designing of the FSR. They should be optimized through numerous EM simulations. The simulation results for the FSR with different resistance value $R_{1}$ are shown in Figure 3. It can be noticed that the influence of the resistance value on the absorbing band is more significant than that on the passband. With the increase of $R_{1}$, the impedance matching of the lower part of absorption band is becoming better, but the impedance matching begins to deteriorate in the upper part of the absorption band. In this respect, an optimal impedance matching can be achieved when the value of $R_{1}$ was chosen as $127 \Omega$. 


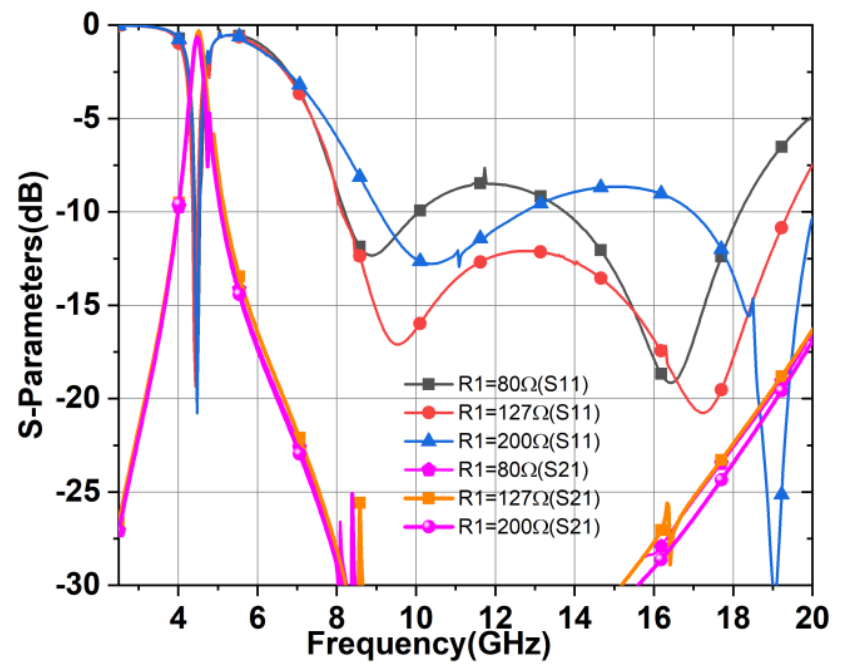

Figure 3. Simulated $S$ parameter with different resistance value of $R_{1}$.

Another important parameter related to the absorption band is the length, $a_{1}$. Changing the value of $a_{1}$ is equivalent to changing the inductance value of $L_{1}$ in the ECM. Figure 4 demonstrates the changing tendency of the performance of the proposed FSR due to different $a_{1}$ values. When $\mathrm{a}_{1}$ increases, the starting frequency of the absorption band will decrease. The reason for the frequency offset is that when the a1 increases, the equivalent inductance becomes larger, and thus the resonant frequency becomes smaller. In addition, it has been shown that with an increase of a1, the absorption performance becomes worse. Considering these effects, an optimal value of $a_{1}$ was achieved at $4.8 \mathrm{~mm}$ in order to obtain the best performance for both in-band and out-of-band signals.

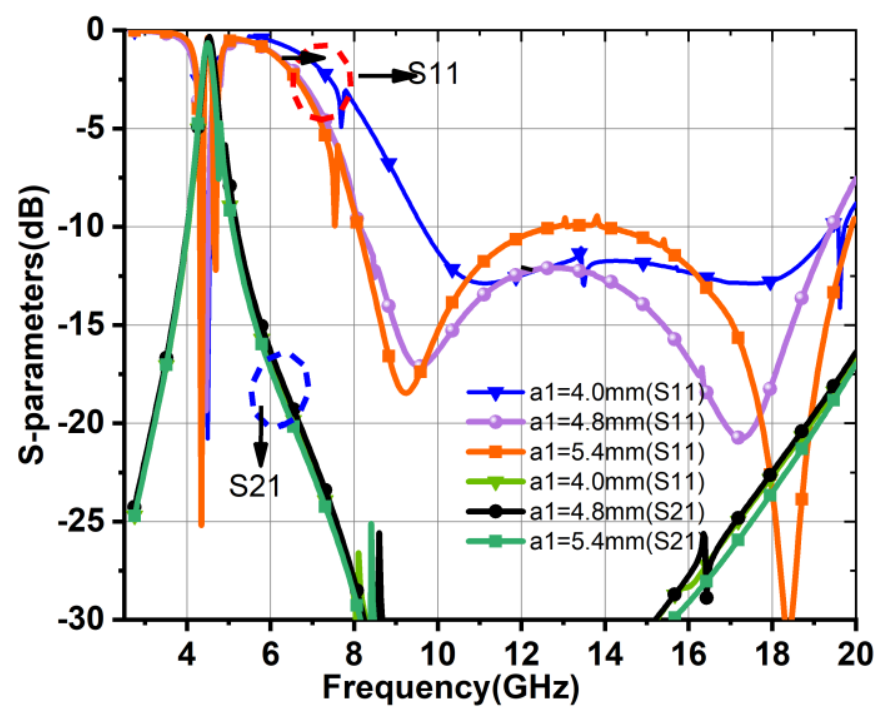

Figure 4. S-parameters with different a1 values.

Furthermore, the surface current distributions of the loss and lossless layers are shown in Figure 5 at three resonances frequency points: $4.5 \mathrm{GHz}, 9.4 \mathrm{GHz}$, and $17.4 \mathrm{GHz}$. The color plot shows that at $4.5 \mathrm{GHz}$, a strong current distribution appeared on the bottom layer whereas the current on the top layer was quite weak. This demonstrates that the two LC-resonators on the $\mathrm{Z}_{1}$ branch of the ECM were in open-circuit states at this frequency. Thus, the FSR acts as a transparent sheet for the incoming EM waves at this frequency. When the frequency of the incident EM waves was 9.4 or $17.4 \mathrm{GHz}$, the surface current distribution feature was opposite to that of $4.5 \mathrm{GHz}$. At 9.4 and $17.4 \mathrm{GHz}$, 
impedances of the LC resonators became inductive or capacitive, respectively. According to ECM, this leads to series resonance on the corresponding circuit branch, and attenuation mainly depends on the resistors. In other words, when the frequency of the incident wave was within the absorption band, most energy was reflected from the lossless layer to the lossy layer, the resistors absorbed most of the incident power, and no resonance occurred on the lossless layer.

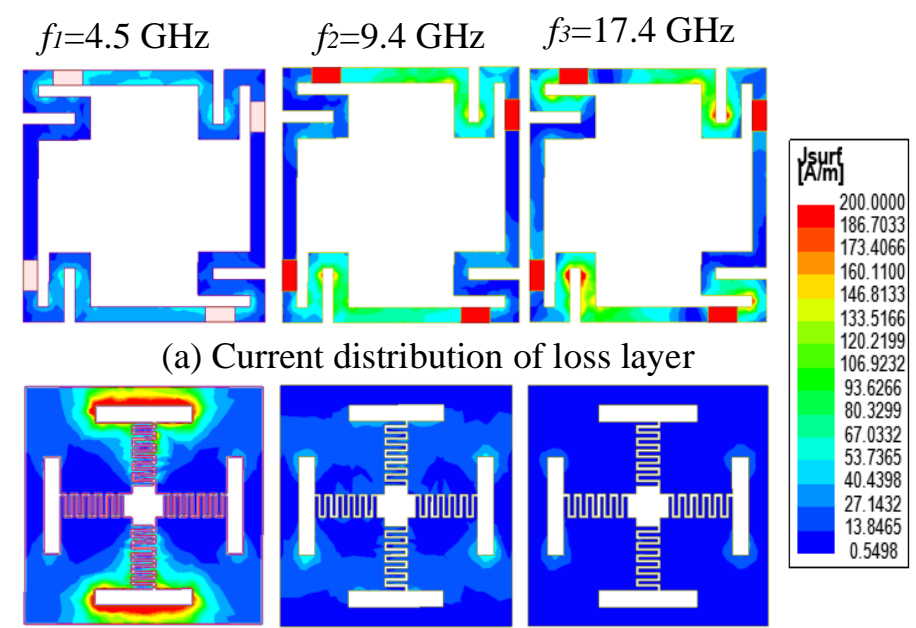

(b) Current distribution of loss layer

Figure 5. Surface current distribution of the lossy layer and lossless layer at $4.5 \mathrm{GHz}, 9.4 \mathrm{GHz}$, and 17.4 GHz.

Since the pattern of the unit cell has C4 symmetry, the proposed design can be used for dual-polarization applications. On the other hand, the minimized unit size will be beneficial for reducing the grating lobes or providing angle stability [23]. In Figure 6a,b, it can be observed that for both TE and TM polarizations, the simulation results of the proposed FSR under different incidence angles did not change much. For the TE polarization, the $10 \mathrm{~dB}$ absorption band ranged from 8.1 to 19.1 GHz, and also showed good angular stability when the incidence angle varies from $0^{\circ}$ to $30^{\circ}$. For the TM polarization, the $10 \mathrm{~dB}$ absorption band shrinks a little bit compared with that of the normal incidence, which extends from 9.3 to $19.1 \mathrm{GHz}$. In short, the performance of the FSR still remained acceptable when the incident angle of the EM waves varied from $-30^{\circ}$ to $30^{\circ}$ due to the compact and symmetric unit cell design.

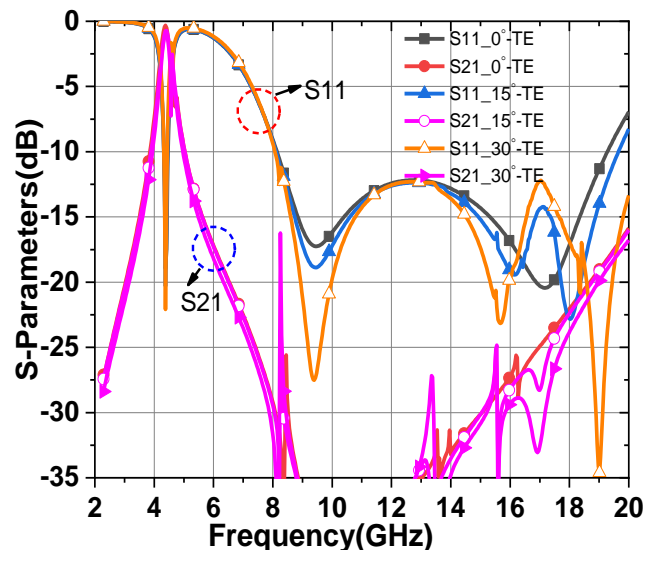

(a)

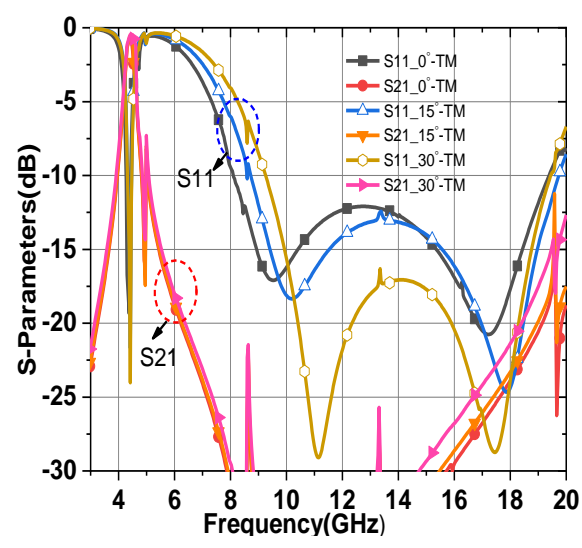

(b)

Figure 6. S parameters of the proposed FSR under different incident angles of TE and TM polarization. (a) TE polarization; (b) TM polarization. 


\section{Experimental Results}

To verify the performance of the design discussed above, a prototype of the proposed FSR was fabricated and measured. Figure 7a shows photographs of our fabricated FSR, which consists of $15 \times 20$ units with a size of $150 \mathrm{~mm} \times 200 \mathrm{~mm} \times 5.1 \mathrm{~mm}$. Two layers of substrate are isolated by nylon bolts and nylon columns to ensure a distance of $3.5 \mathrm{~mm}$ between both substrates.

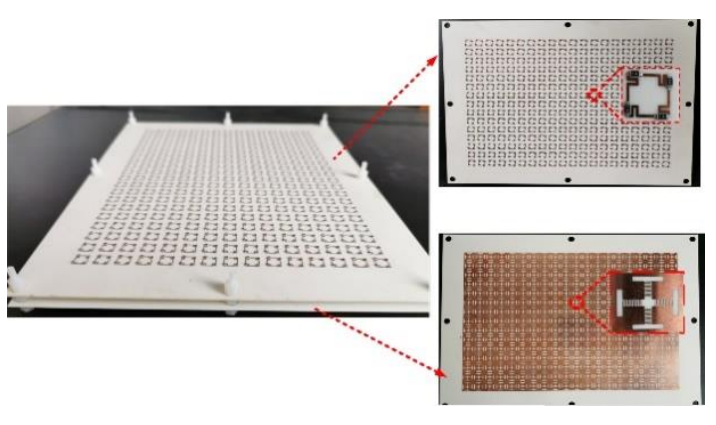

(a)

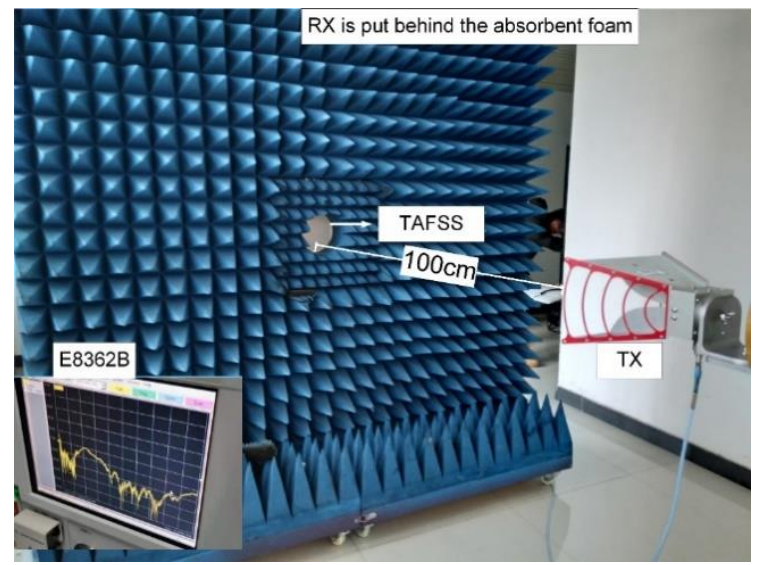

(b)

Figure 7. Photographs of the fabricated FSR. (a) Top view of FSR; (b) measurement setup of the FSR.

The measurement setup of the proposed FSR is shown in Figure $7 \mathrm{~b}$. The fabricated prototype was embedded into the absorbing screen, which was placed between the transmitting horn and receiving horn antenna. The vector network analyzer Agilent E8362B was used to measure the S parameter. It can be clearly observed that the measured results agreed quite well with the simulation, as is illustrated in Figure 8. The measurement results showed that the transmission band was located at $4.5 \mathrm{GHz}$ with an IL of $0.45 \mathrm{~dB}$. On the other hand, we achieved an absorption band that extended from 8.1 to $19 \mathrm{GHz}$. The slight differences between the simulation and measurement may be due to the inaccuracy of the air spacer's thickness and manufacturing error. Alternatively, it is worth noting that the amplitude fluctuation of the measured results was mainly caused by the coupling and interaction between the two horn antennas and the diffraction of electromagnetic waves.

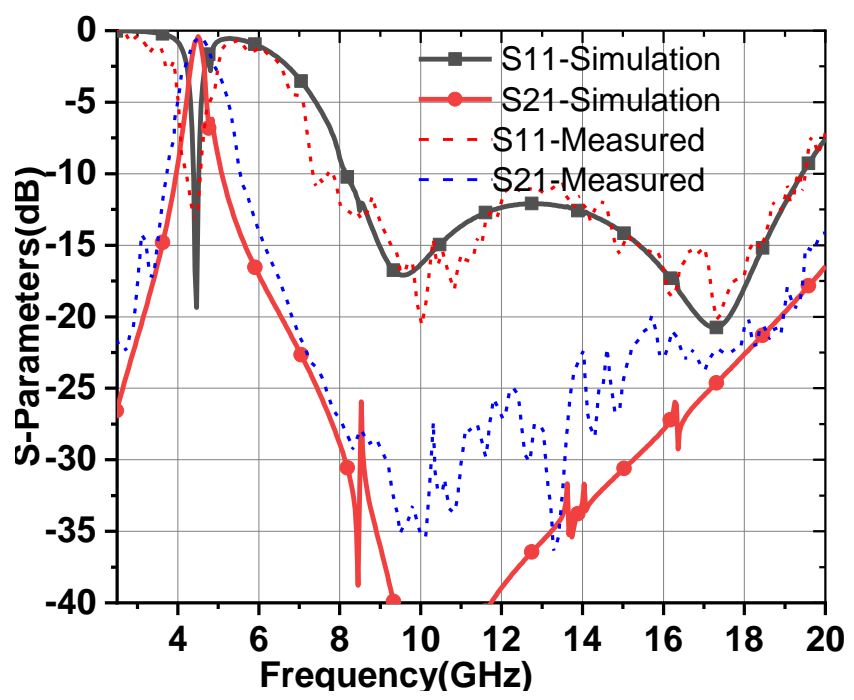

Figure 8. S parameter of the simulation and the measurement results under normal incidence. 
To illustrate the advantages of the proposed design, we performed a comparison between our design and other published studies in terms of transmission band, insertion loss, $10 \mathrm{~dB}$ absorption bandwidth, thickness, cell size, and absorptive/transmissive performance, which is shown in Table 2. The proposed FSR in this work has better transmission, a lower profile, and a broader $10 \mathrm{~dB}$ absorption band that covers both the X-and Ku-bands.

Table 2. Comparison between the proposed FSR and others presented the literature.

\begin{tabular}{cccccc}
\hline Ref. & $\begin{array}{c}\text { Transmission Band } \\
(\mathbf{G H z}) / \mathbf{I L}(\mathbf{d B})\end{array}$ & $\begin{array}{c}\text { 10 dB Absorption } \\
\text { Band (GHz)/FBW }\end{array}$ & Thickness & Periodicity & Form \\
\hline$[11]$ & $1.6 / \mathrm{NA}$ & $5.3-14.8 / 95 \%$ & $4.5 \mathrm{~mm}\left(0.08 \lambda_{\mathrm{a}}{ }^{2}\right)$ & $0.17 \lambda_{\mathrm{a}} \times 0.17 \lambda_{\mathrm{a}}$ & $2 \mathrm{D}$ \\
{$[21]$} & $0.915 / \mathrm{NA}$ & $3.93-8.07 / 69 \%$ & $9.5 \mathrm{~mm}\left(0.15 \lambda_{\mathrm{a}}\right)$ & $0.12 \lambda_{\mathrm{a}} \times 0.12 \lambda_{\mathrm{a}}$ & $2 \mathrm{D}$ \\
{$[22]$} & $3.4 /-1.2$ & $11-18 / 47 \%$ & $4.75 \mathrm{~mm}\left(0.18 \lambda_{\mathrm{a}}\right)$ & $0.3 \lambda_{\mathrm{a}} \times 0.3 \lambda_{\mathrm{a}}$ & $3 \mathrm{D}$ \\
{$[23]$} & $4.15 /-2.4$ & $4.8-9.3 / 64 \%$ & $4.67 \mathrm{~mm}\left(0.07 \lambda_{\mathrm{a}}\right)$ & $0.31 \lambda_{\mathrm{a}} \times 0.31 \lambda_{\mathrm{a}}$ & $3 \mathrm{D}$ \\
{$[24]$} & $4.6 /-0.3$ & $10-18 / 57 \%$ & $5 \mathrm{~mm}\left(0.17 \lambda_{\mathrm{a}}\right)$ & $0.55 \lambda_{\mathrm{a}} \times 0.55 \lambda_{\mathrm{a}}$ & $2 \mathrm{D}$ \\
{$[25]$} & $1.5 / \mathrm{NA}$ & $4.95-8.07 / 34 \%$ & $8 \mathrm{~mm}\left(0.13 \lambda_{\mathrm{a}}\right)$ & $0.15 \lambda_{\mathrm{a}} \times 15 \lambda_{\mathrm{a}}$ & $2 \mathrm{D}$ \\
{$[26]$} & $1 /-0.6$ & $3.2-8.8 / 93 \%$ & $7.5 \mathrm{~mm}\left(0.08 \lambda_{\mathrm{a}}\right)$ & $0.1 \lambda_{\mathrm{a}} \times 0.1 \lambda_{\mathrm{a}}$ & $2 \mathrm{D}$ \\
{$[15]$} & $2.45 /-1.2$ & $7.68-21.7 / 95 \%$ & $8 \mathrm{~mm}\left(0.2 \lambda_{\mathrm{a}}\right)$ & $0.13 \lambda_{\mathrm{a}} \times 0.13 \lambda_{\mathrm{a}}$ & $2 \mathrm{D}$ \\
This work & $4.5 /-0.45$ & $8.1-19.1 / 81 \%$ & $3.5 \mathrm{~mm}\left(0.09 \lambda_{\mathrm{a}}\right)$ & $0.27 \lambda_{\mathrm{a}} \times 0.27 \lambda_{\mathrm{a}}$ & $2 \mathrm{D}$ \\
\hline
\end{tabular}

${ }^{1}$ NA means the data in the paper are not given; ${ }^{2 \cdot} \lambda_{\mathrm{a}}$ is wavelength at the lowest frequency of the absorption band.

\section{Conclusions}

In this paper, a novel dual-polarized FSR with ultrawide absorption spectrum covering both the $\mathrm{X}$ - and $\mathrm{Ku}$-bands was proposed. A Jerusalem cross aperture array filled with a meanderline in vertical cross-slots is used to generate the passband and reduce unit size. Its small period size avoids the generation of grating lobes in the absorbing band and the angular stability can be maintained. To minimize the insertion loss, a specially designed incurved square loop loaded with resistors was chosen as the absorbing unit cell. An equivalent circuit model was provided to explain the operating principle. The designed FSR unit has a minimized size of $0.27 \lambda_{a} \times 0.27 \lambda_{a}$, and the FSR also keeps a lower profile with a total thickness of $5.1 \mathrm{~mm}$, i.e., $0.13 \lambda_{\mathrm{a}}$. The absorptive band width spans from 8.1 to $19.1 \mathrm{GHz}$, and the passband can be obtained at $4.5 \mathrm{GHz}$ with an insertion loss of only $0.45 \mathrm{~dB}$. Both simulation and measurement results agreed well with each other. The novel FSR will have wide applications in the radar stealth of modern military equipment.

Author Contributions: H.L. and J.X. conceived and designed the experiments; J.X. and Y.W. designed the simulations; Y.W. and Y.M. analyzed the data; F.D. and L.C. contributed analysis tools; H.L. and J.X. revised the paper. All authors have read and agreed to the published version of the manuscript.

Funding: This research was funded by the fundamental Research Funds for the Central University of China under grant "CCNU18JCXK02, CCNU18GF006, CCNU16A02016, CCNU19TS073."; the open fund of Guangxi Key Laboratory of wireless wideband communication and Signal Processing under grant "GXKL06190202"; the open fund of China Ship Development and Design Centre under grant "XM0120190196"; funded in part by the Beijing Orient Institute of Measurement and Test Electrostatic Research Foundation of Liu Shanghe Academicians and Experts Workstation under Grant BOIMTLSHJD20181002.

Acknowledgments: We would like to thank Feng Yijun and Zhao Junming of Nanjing University for providing the measurements setup.

Conflicts of Interest: The authors declare no conflict of interest.

\section{References}

1. Ohta, H.; Lang, K.; Tsao, C.; Mittra, R. Frequency selective surface for satellite communications antenna applications. Antennas Propag. Soc. Int. Symp. IEEE 1982, 475, 478.

2. Sarabandi, K.; Behdad, N. A Frequency selective surface with miniaturized elements. IEEE Trans. Antennas Propag. 2007, 55, 1239-1245. [CrossRef] 
3. Krushna Kanth, V.; Raghavan, S. Em design and analysis of frequency selective surface based on substrate-integrated waveguide technology for airborne Radome application. IEEE Trans. Microw. Theory Tech. 2019, 67, 1727-1739. [CrossRef]

4. Yan, Q.; Sun, Y.; Yue, D.W. LOS-based equal gain transmission and combining in general frequency-selective Ricean massive MIMO Channels. Electronics 2019, 8, 79. [CrossRef]

5. Dai, H.; Zhao, Y.; Li, H.; Chen, J.; He, Z.; Qi, W. An ultra-wide band polarization-independent random coding Metasurface for RCS reduction. Electronics 2019, 8, 1104. [CrossRef]

6. Bakshi, S.C.; Mitra, D.; Ghosh, S. A Frequency Selective Surface Based Reconfigurable Rasorber with Switchable Transmission/Reflection Band. IEEE Antennas Wirel. Propag. Lett. 2018, 18, 29-33. [CrossRef]

7. Motevasselian, A.; Jonsson, B.L.G. Design of a wideband rasorber with a polarisation-sensitive transparent window. IET Microw. Antennas Propag. 2012, 6, 747-755. [CrossRef]

8. Sheokand, H.; Singh, G.; Ghosh, S.; Ramkumar, J.; Ramakrishna, S.A.; Srivastva, K.V. An Optically Transparent Broadband Microwave Absorber using Interdigital Capacitance. IEEE Antennas Wirel. Propag. Lett. 2018, 18, 113-117. [CrossRef]

9. Arceneaux, W.S.; Akins, R.D.; May, W.B. Absorptive/Transmissive Radome. U.S. Patent 5400043, 1995.

10. Maxwell, S. Radar Absorptive Coating. U.S. Patent 3599210, 1971.

11. Chen, Q.; Liu, L.; Chen, L.; Bai, J.; Fu, Y. Absorptive frequency selective surface using parallel LC resonance. Electron. Lett. 2016, 52, 418-419. [CrossRef]

12. Yu, J.; Jiang, W.; Gong, S. Wideband Angular Stable Absorber Based on Spoof Surface Plasmon Polariton for RCS Reduction. IEEE Antennas Wirel. Propag. Lett. 2020, 19, 1058-1062. [CrossRef]

13. Wei, P.-S.; Chiu, C.-N.; Wu, T.-L. Design and analysis of an ultraminiaturized frequency selective surface with two arbitrary stopbands. IEEE Trans. Electromagn. Compat. 2019, 61, 1447-1456. [CrossRef]

14. Zhang, B.; Jin, C.; Shen, Z. Absorptive frequency selective reflector based on bent metallic strip embedded with chip-resistors. IEEE Trans. Antennas Propag. 2020, 68, 5736-5741. [CrossRef]

15. Wang, Y.; Qi, S.-S.; Shen, Z.; Wu, W. Ultrathin 3-D Frequency Selective Rasorber with Wide Absorption Bands. IEEE Trans. Antennas Propag. 2020, 68, 4697-4705. [CrossRef]

16. Singh, D.; Yadav, R.P. A 3-D printed square loop frequency selective surface for harmonic radar applications. J. Electromagn. Waves Appl. 2020, 34, 396-406. [CrossRef]

17. Yu, Y.; Luo, G.Q.; Yu, W.; Tong, J.; Shen, Z. 3d Frequency Selective Rasorber Based on Magnetic Material and Meander-Line. IEEE Trans. Antennas Propag. 2020, 1. [CrossRef]

18. Omar, A.A.; Huang, H.; Shen, Z. Absorptive Frequency selective Reflection/Transmission Structures. IEEE Antennas Propag. Mag. 2020, 62, 62-74. [CrossRef]

19. Yi, B.; Liu, P.; Li, G.; Dong, Y. Design of miniaturized and ultrathin absorptive/transmissive radome with wideband absorbing property. Microw. Opt. Technol. Lett. 2016, 58, 1870-1875. [CrossRef]

20. Li, H. Design and analysis of a frequency selective radome (FSR) with wideband absorbing properties. In Proceedings of the IEEE International Workshop on Electromagnetics: Applications \& Student Innovation Competition, Nanjing, China, 16-18 May 2016.

21. Liu, L.; Zhai, H.; Hu, D.; Ren, S. A polarization-stable frequency selective rasorber with miniaturized elements and wideband absorbing properties. Microw. Opt. Technol. Lett. 2020, 62, 1643-1650. [CrossRef]

22. Yu, W.; Luo, G.Q.; Yu, Y.; Zhang, X.H.; Fan, K. Miniaturized band-absorptive frequency selective rasorbers with wide absorption band. IET Microw. Antennas Propag. 2019, 13, 1777-1781. [CrossRef]

23. Hu, D.; Zhai, H.; Liu, L.; Shi, J.; Nie, Z. A new miniaturized frequency selective surface designed for Ku-band absorption and low-frequency bandpass. Microw. Opt. Technol. Lett. 2020, 62, 315-321. [CrossRef]

24. Li, B.; Shen, Z. Wideband 3D Frequency Selective Rasorber. IEEE Trans. Antennas Propag. 2014, 62, 6536-6541. [CrossRef]

25. Costa, F.; Monorchio, A. A Frequency Selective Radome with Wideband Absorbing Properties. IEEE Trans. Antennas Propag. 2012, 60, 2740-2747. [CrossRef]

26. Yu, W.; Luo, G.Q.; Yu, Y.; Pan, Y.; Shen, Z. Dual-Polarized Band-Absorptive Frequency Selective Rasorber Using Meander-Line and Lumped Resistors. IEEE Trans. Antennas Propag. 2019, 67, 1318-1322. [CrossRef]

(C) 2020 by the authors. Licensee MDPI, Basel, Switzerland. This article is an open access article distributed under the terms and conditions of the Creative Commons Attribution (CC BY) license (http://creativecommons.org/licenses/by/4.0/). 